\title{
The Handle of Substation Remote Terminal Unit Configuration
}

\author{
Igor Dmitrenko, Tallinn University of Technology, Elering OÜ, Juhan Laugis, Tallinn University of Technology
}

\begin{abstract}
The remote control and supervision of electrical power transmission system is the balanced system between electricity generating and consuming, taking the responsibility for the stability of power supply consuming. The device to be responsible for above-mentioned tasks is a Remote Terminal Unit with faultless proper configuration.
\end{abstract}

Keywords: communication terminal, testing, SCADA systems, software.

\section{INTRODUCTION}

The goal of work is to differentiate the circumstances following the delivery, maintaining, parameterization and commissioning of Remote Terminal Unit (RTU) in the main grid substation. The solution how to hold the control on proper functionality of RTU during its whole life cycle taking into consideration possible modification of main grid substation telematics system.

The basic data and materials for current analysis are kindly given to represent by Estonian transmission system operator (TSO) company Elering OÜ.

Elering OÜ (National Grid, Main Grid) is Estonian transmission system operator (TSO). Using 110-330 kV highvoltage power lines, it unites Estonia's biggest power stations, distribution networks and corporate consumers in an integral energy system. Elering OÜ has international grid connections, hence the Estonian TSO is a member of several international organizations, engaged in power transmission (see "Fig. 1").

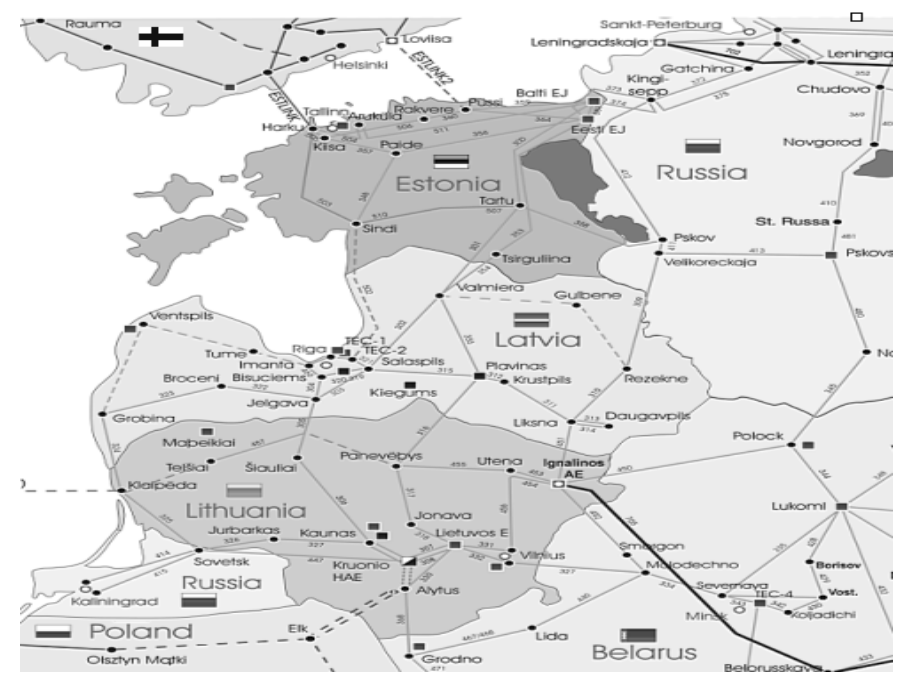

Fig. 1. Position of Estonian electrical power transmission system on map.
The main functions of Elering OÜ are listed below:

- ensuring the energy market existence according to European Union regulations;

- transmitting electricity at voltages of $6-330 \mathrm{kV}$ to distribution networks and corporate consumers;

- ensuring the reliability of Estonia's electrical power system in conjunction with the power systems of the neighboring countries (see "Fig. 1");

- maintaining the capacity balance of the electrical power transmission system and managing the power system across Estonia in real time;

- ensuring the Estonian energy balance and operating the balance settlement for the balance providers.

\section{OVERVIEW OF TELEMATICS IN POWER TRANSMISSION SUBSTATION}

The main parts of telemechanical control and supervisory for power grid are Remote Control Centre (RCC), RTU, IEDs (Intelligent Electronic Devices), measurement transducers, local reference clock device and communications paths.

The substation RTU is device for immediate reaction on information objects (control, status, measurement messages), receiving them from side to remotely connected RCC Supervisory Control and Data Acquisition system (SCADA system). On the local side the RTU shall be connected to the executing device, that reacts on control or parameter set action from SCADA system personnel.

The reliability of the substation RTU during its lifecycle and the parameterization for proper working according to SCADA system operating needs should be ensured by technical requirements and by continuous checking of readyto-act substation apparatus.

Usually the primary equipment of substation should be connected to the IED, that tasks are local control and monitoring of primary circuit apparatus, protection function and the providing of remote control and monitoring opportunities. Very seldom in case of the IEDs are not requested, the only substation RTU has responsibility on all above-mentioned functions.

The measurement transducer is the device, that sends the operational measurement data to the SCADA system via the RTU.

Nowadays the most popular physical way to ensure the reliable data exchanging between protection relay and RTU is optical data transmission, i.e. using of optical cables. 
As a rule the data transmission protocol IEC 60870-5-101 (serial) and/or IEC 60870-5-104 (TCP/IP) is the low and upper level of data transmission between substation equipment and the SCADA system. The data transmission protocol IEC 60870-5-103 (serial) is used for connecting different independent devices (e.g. IEDs) or their groups to the RTU. The mostly used data transmission protocol standards like Modbus, Profibus are well-known solutions for communication between measurement transducers and the RTU. The one of the important SCADA system requirements is the time synchronizing of whole substation telematics. The principle structure of is shown on "Fig. 2".

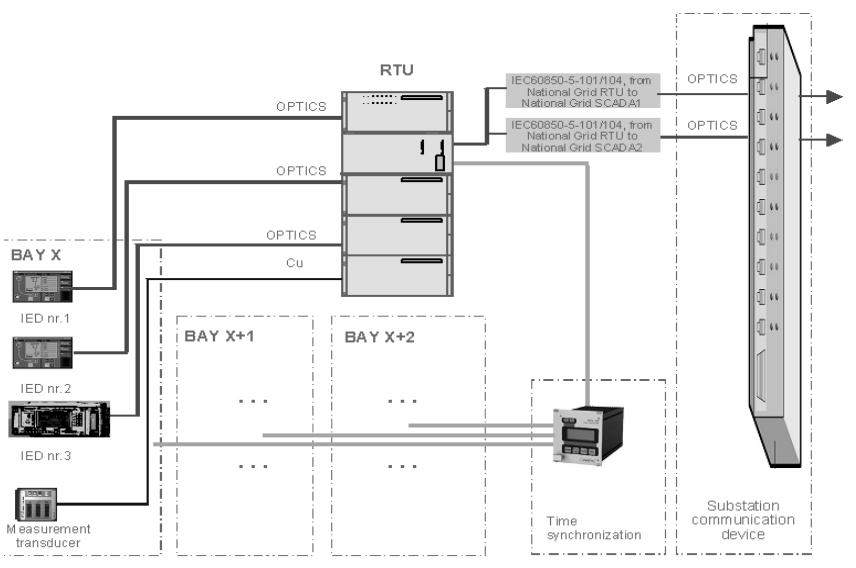

Fig. 2. Simplified structure of bay telematics.

The purpose of substation reference clock with appropriated input signal (e.g. Global Position System signal) or and time synchronizer is dedicated for correct real local time and date run in IED-s (incl. RTU) and for correct time stamp of signal, that is sent to the SCADA system.

\section{THE REALIZING OF THE TELEMATICS PART IN THE PROJECT}

The construction of new substation telematic system presumes some steps made before. There are some of them:

- necessity to build the new telematics system in substation;

- budgetary possibilities for building of the new telematics system in substation;

- technical requirements for telematics system in scope of supply;

- the professional qualification of Contractor by Client;

- signed procurement with suitable Contractor for telematics works defined in scope of supply works;

- existence of Client's project workgroup with appropriated "know-how".

The realization method and management style for project accomplishment could be different, where a few of abovementioned points would be skipped.

\section{A. Contractual method, "turn-key"}

This realization method of project is necessary in case of Client insufficient or lack of needed specialists, materials, building techniques etc, that are irreplaceable for successful finalizing of preplanned project or project part. There should be well developed relationships between Client and Contractor during the tender. The both Client and Contractor set up his own workgroup with their own project managers.

Common benefits:

- more than one project could be managed by Client, while the Contractors may be different and independent;

- the responsibility for uncompleted project lies with Contractor;

- the Client accepts only finished building object, i.e. the substation.

- the Client accepts only finished building object, i.e. the substation.

Common drawback:

- continuous Client check the accordance of Contractor technical solutions to Client requirements.

\section{B. "Self-made" method}

This realization method of project is opposite to previous. The main idea of "self-made" method is to reduce the project costs using own personnel, knowledge, materials, mechanics etc.

Common benefits:

- cost reducing of the project;

- timesaving for some internal agreement during the project;

- flexibility for realizing the project.

Common drawbacks:

- the company should have capacities to cover all of activities concerning to realizing of the project. I.e. planning, drawing, working personnel qualification with valid licenses and certification, bulk sales contacts, machinery park;

- long-term duration of realizing the project.

\section{Split to multitask method}

As a rule the project consists from several work tasks. Some of them could be established internally by the workforce of the project initiator. But for some work tasks, to be accomplished by internal execution, it is not really purposeful, because the expense for company internal realizing compared to outsourcing is prevalent. Hence splitting the work to multitask could be very rational in such case.

Common benefit:

- reduce the price of the project by splitting the work to multitask scope. 
Common drawback:

- the part of Client work scope and Contractor work scope shall be clearly specify during the project planning stage.

\section{THE PRINCIPLE OF RTU CONFIGURATION COMPOSING}

The main parts of the RTU are:

- the hardware (rack, power supply module, processor module, I/O-modules, communication modules etc);

- the firmware (small program to control internally different components of hardware module);

- the parameterization;

- the communication links for external telematics consumers (different networks, cables, communication devices for data exchanging with SCADA system, IEDs, measurement transducers, GPS time synchronization system etc).

The select of RTU hardware components should be done according to technical requirements stated in quality documents of Client.

The RTU software affords all parameterization features, that enable to perform all needed RTU adjustments. As a rule the parameterization features of RTU configuration software are divided to four groups:

- hardware group;

- communication interfaces with external telematics devices;

- logic function group;

- group of information objects exchange with telematics system participants.

The RTU hardware should be correctly defined by RTU software. The reason of that, in addition to the location of different RTU extension modules for their proper using in couple with external telematics devices, is the ensuring of internal communication between RTU modules via system bus.

The communication interfaces of RTU support all possible RTU connections with SCADA system, IEDs, measurement transducers and GPS time synchronization system.

The logic realizing requires also using of the standard logical elements like AND, OR, NOR and/or some extended block-elements e.g. measurement converter in RTU. The grouping of some sub-signals with following composing the independent group-signal in RTU for SCADA system belongs to logic function too.

The most obstructive part of the RTU parameterization is the definition of signals (information objects) transmitted and received by the RTU. The ground of that is the adjustment of various number of information objects parameters.

The basic principles of RTU parameterizing handle is shown on figure "Fig. 3".

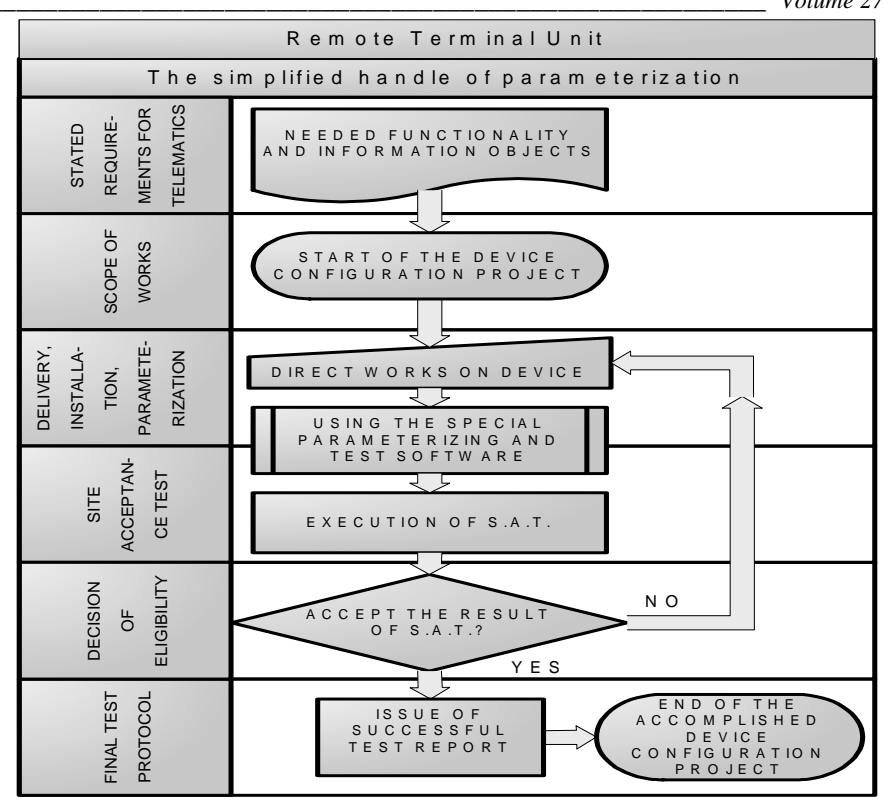

Fig. 3. Simplified handle structure of the RTU parameterization.

The most important document describes the needed telematics system is the Client's requirements statement. This document is mostly concentrated on specification of the RTU requirements, but also depends on some requirements stated for another telematics devices (e.g. for measurement transducer). The necessity of the valid RTU requirements becomes obvious in:

- the RTU compatibility to the existing RCC equipment;

- the RTU compatibility to the new and/or existing substation equipment;

- the transparency of the telematics system with RTU for supervision and future modification;

- standardized solutions of the telematics system equipped with RTU, i.e. use of international standards by constructing and commissioning of the telematics system;

- easy discover of the possible failure in the RTU;

- easy replacement of failed RTU modules;

- independent reparation of the possible failed RTU modules from others proper working;

- etc.

There is another inseparable part of supply documents that helps to make precise the features and functions of the RTU comparing to common requirements. Its name is the scope of supply document, that illustrates more or less the actual demands for specified substation RTU. Hence the Contractor shall obey requirements stated in both documents.

The parameterization of RTU is making by RTU specialist using a usual human-machine interface (keyboard, mouse, display) of the PC. Each manufacturer uses his own RTU software for his RTU parameterization. There are a lot of different RTU software tools that equipped with specific features of human-machine interface (HMI). In result of this, there are many possibilities to present the image of the RTU internal structure in configuration software. To make it the 
RTU software easily to understand for the Client personnel it should meet requirements of IEC standard compatibility, e.g. the international standard IEC 61131 for programmable logic controllers (e.g. part 3. programming languages: ladder diagram, sequential function charts, function block diagram, structured text, instruction list). Thus the data types, objects, function blocks, logic etc defined by RTU software do not confuse Client personnel in spite of software manufacturer.

If whole or part (substation extension) of RTU parameterization is ready for implementation, the preliminary test of RTU parameterization will be established to detect the possible RTU internal mistake or conflict. It will be performed using several testing software programs. There are two solutions for that:

- use implemented in RTU parameterization software check functions (e.g. "compile" or "consistency check");

- use external software to test end results of RTU configuration (e.g. IEC 60870-5-101 data transmission protocol test system).

Of course, the first method is more preferable and less cost method to test the made RTU configuration. The second method involves third-part testing software like RTU parameterization software.

After above mentioned testing processes the site acceptance test (SAT) of RTU configuration belongs to fulfill. The SAT is the one of routine tests for the telematics systems that are commonly:

- design test (manufacture's test);

- site acceptance test (SAT and pre-SAT; on-site device test before it's final commissioning);

- special test (according to the necessity for Client).

The functional target of RTU should be checked by the execution of the SAT. As a rule there are more than one telematics device connected to the RTU, that should react adequately to RTU behavior. Hence in case of precursory RTU parameterization, the telematics system participants like IEDs (e.g. protection relays) should be also parameterized correctly.

It needs to be careful that single modification of some parameters in telematics participant device will cause a failure because of non-up-to-date RTU parameterization. In the best case it will end with failure of some remote control functions for SCADA system. The worst case will cause the human accident like remote high voltage energizing operation of power overhead line servicing by maintenance personnel.

The best solution of SAT execution is not only to test all the parameterized information objects defined in RTU, like their test from "lower" level (IEDs binary inputs, RTU binary/analog inputs etc) up to the SCADA system, including the testing of all remote commands execution from RCC. Thus the RTU configuration process includes more steps concerning the other telematics devices (figure "Fig. 4").

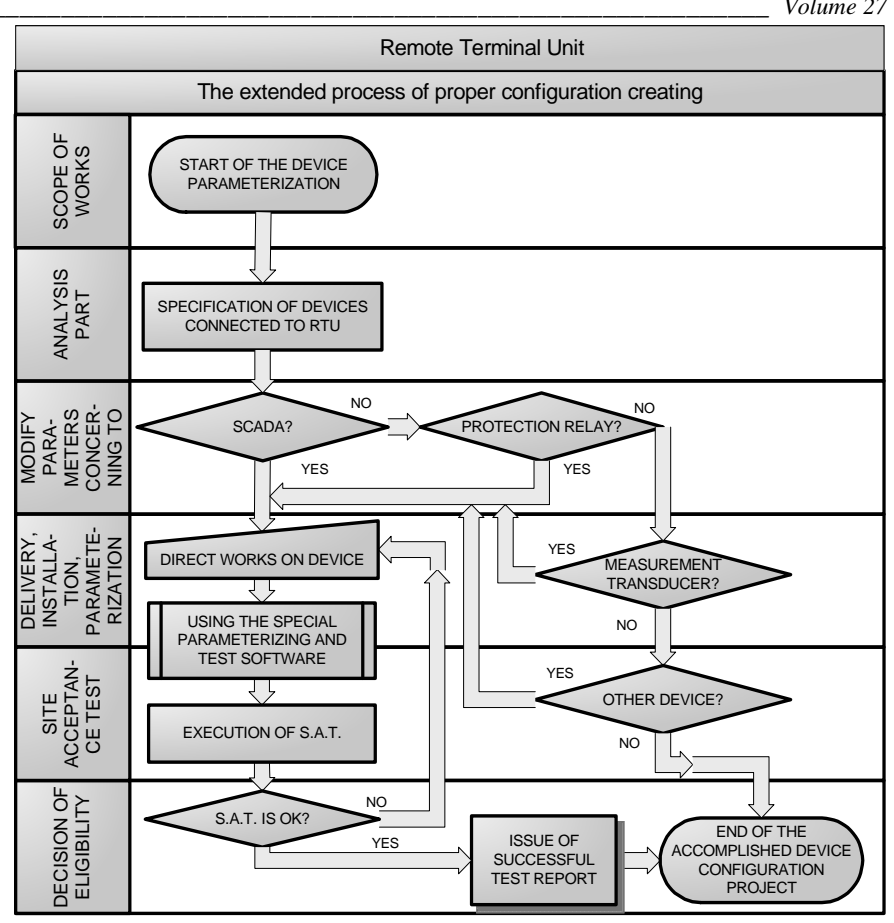

Fig. 4. The extended structure of RTU configuration process.

There should be no any mitigation to reduce the testing amount of information objects even concerning logic grouping of information objects composed in RTU or IED. All subinformation objects belong to separate SAT performance.

The above described handle process scenario of the RTU parameterization should be valid not only by new RTU placement and its parameterization works, but also in case of any extension of substation telematics system.

\section{V.THE FINALIZATION OF THE RTU WORKS. HINDRANCES}

When all above defined steps of the RTU configuration project are successfully performed and RTU is already put into continuous failure-free operation, the handover process of the project documentation, secondary device (telematics, protection, communication etc) parameterization files and configuration software should be started.

There are three possibilities of backup the RTU parameterization files with appropriated (used for making RTU parameterization) software:

- get the RTU parameterization files and RTU software to keep and use it by Client;

- get the RTU parameterization files and RTU software. To conclude a contract with external company, that will take the responsibility for keeping and handling over of the appropriated RTU parameterization files and RTU software; - to conclude a contract with Contractor or manufacturer, that will ensure keeping and handling over of the appropriated RTU parameterization files and RTU software.

The first variant is mostly used by power transmission companies that are able to manage substation building project and construct the substation within own company. But it is 
also common for companies, that intend to carry through the "turn-key" projects.

The second one is more suitable for companies that do not have technical personnel to service the secondary substation devices.

The last variant is rarely used because of needs to have a special relationship between the Contractor and Client with possible reciprocal company ownership.

There are some clearly distinguished parts of RTU software and needed accessories:

- RTU software ownership licences;

- RTU software installation package;

- PC/RTU communication adapter for parameterization, cable etc (optional).

There are three different types of licence for RTU software depending on manufacturer:

- paper based document (written whom is licenced, licence number, product code etc);

- data fail licence (needed for proper working of RTU software);

- external data storage unit (e.g. CD-ROM);

- external licence holding device (hardware lock, dongle; needed for starting and working of RTU software).

Some manufacturer of RTU software uses all types of above listed licences at once. But someone has also RTU hardware dongles (e.g. IEC 60870-5-101 communication dongle) despite already existing licences for RTU software. This kind of dongle should be attached to the RTU hardware for proper working of RTU communication.

When running status of the RTU will be fixed and assured then all of those materials will be delivered from Contractor to the Client. Thus the Client needs to be more careful and well informed about content of certain RTU delivery proposal.

The one reason should be noticed, that continuously developing technology of RTU in hardware part and in software could cause some "mismatches". That's why the some range of product is increasing rapidly "forgetting" the software support of older device models, that are still in use by Client or even under manufacturing.

Usually the developing of RTU software does not stop if product is ready. The software mistakes, bugs etc will be repaired by extracting of software updates, hot-fixes. Thus such components of RTU software like parameterization features, RTU operational system, RTU firmware etc could also be changed by Contractor during the substation building project. But at the end of project the Client will receive only new RTU software, probably without any additional update, hot-fix or similar. Commonly the newer RTU software enables more functional possibilities for RTU, contains some program corrections, updated firmware, faster processing etc, but it is useless while the lack of RTU support remains for those additional features by older RTU hardware type.
The next unexpected circumstance is possible noncompatibility of the RTU software older version with newer one or impossibility to have both versions of RTU software simultaneously, installed on same Client's PC hard disk.

\section{THE PROPOSAL SOLUTION FOR KEEPING QUALITY CONTROL OF THE RTU}

The localizing of the main key points in amount of needed materials, that belong to request from Contractor, guarantees the proper work of Client's substation RTU:

- proper parameterization of entire RTU;

- flawless definition of information objects concerning to telematics "lower" level (e.g. IEDs);

- flawless definition of information objects concerning telematics "upper" level (RCC, local workstation, time synchronization system);

- flawless definition of logic tasks in the RTU;

- proper parameterization of the RTU related telematics devices;

- appropriated RTU software including all needed service packs, hot-fixes, update, upgrade etc;

- appropriated parameterization adapter needed between RTU and PC with installed RTU software;

- documentation (schemes, manuals, test protocols etc).

Even regardless of some modern methods for present of information object in RTU like MS Excel standard sheet im/exporting, there are still possibility for human factor. Usually the MS Excel sheets with specified information objects belong to conformation by Client (different modification is possible) and after that, the agreed by Client information objects should be parameterized in RTU at once. Unfortunately the RTU parameterization upload to the RTU is not one-time action, but repetitive depending on phase of substation construction project, when partially commissioning of primary devices is necessary (e.g. case of large substation with several voltage level switchyards). Hence the parameterization of the RTU remains still in progress and is daily changing.

If after each modification of RTU parameterization the additional full SAT should be carried out then performing of "intermediate" SAT does not have any sense. The only last SAT on the end of substation construction project (RTU part) could be reliable for setting of RTU test protocol document.

In result of that, the best variant to avoid the human failures by any extension of substation RTU in the future is to demand new independent RTU for extension part of substation.

The important Client idea is to be sure in reliability of proper RTU parameterization files and delivered RTU software. When the all RTU parameterization files are ready for upload to the substation RTU, the Contractor shall declare the finished work and readiness for SAT execution before commissioning according to the method, that is represented on the figure "Fig. 5". At the same time the all other telematics participants shall be ready for SAT too. 


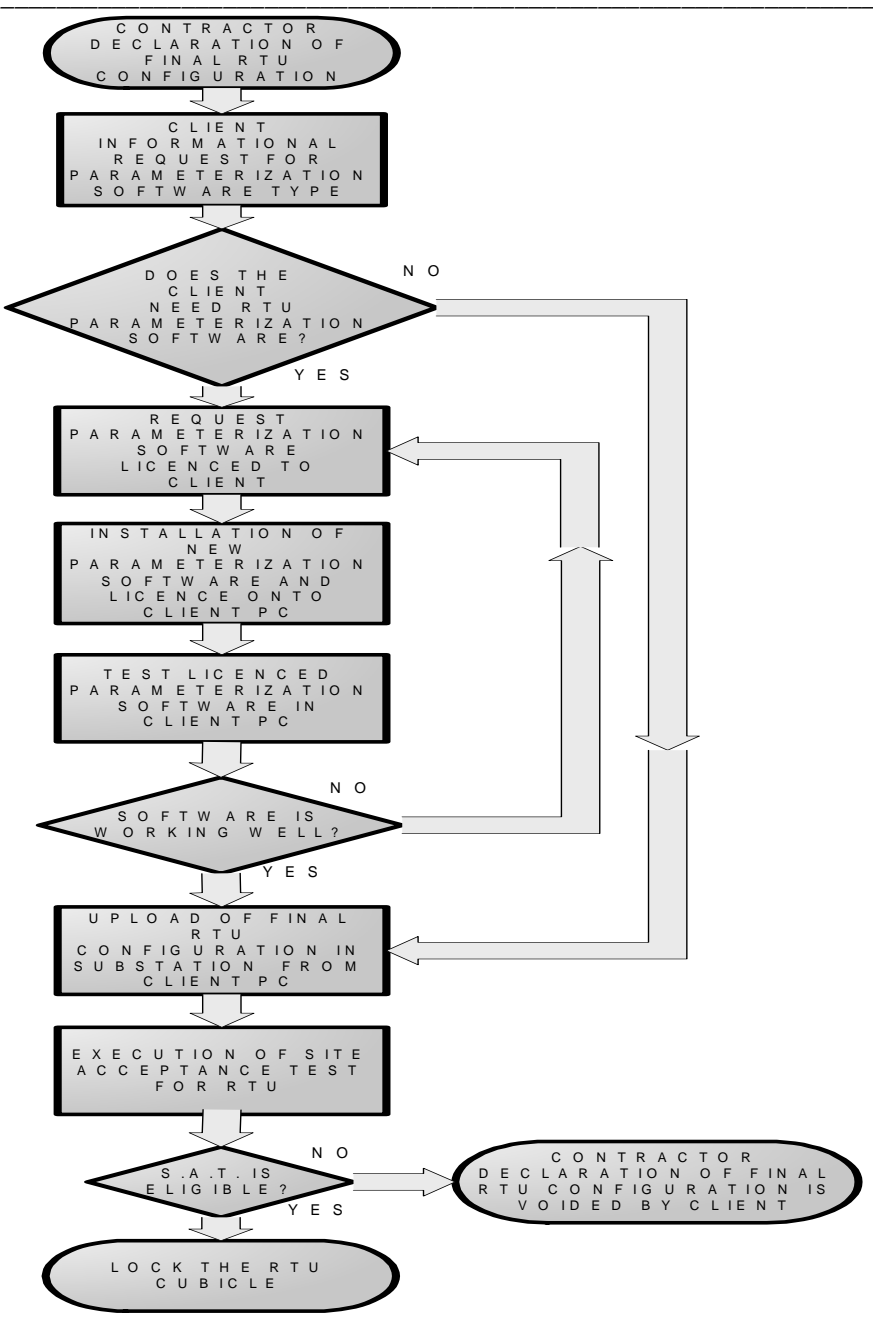

Fig. 5. The check process structure of the RTU.

The additional task for the Contractor is to prepare RTU software, RTU parameterization accessories in PC of the Client. The Contractor should check the version of RTU software with or without extra service packs, hot-fixes etc. In result of Client decision the Contractor shall deliver the full RTU software package with al needed additional updates and upgrades. If the Client needs for new RTU software, the Contractor should deliver it and help him to install it on the Client's PC. Sometimes the extra program licence should be installed for the flawless RTU software running.

After the check of RTU software correct working on Client's PC, the transfer of the RTU parameterization files would be performed from Contractor's PC to the Client's PC. By successful accomplishing of the appropriated RTU files transmission to the Client's PC, the upload of RTU parameterization files will start from Client's PC to the RTU by supervision of Contractor. If it is done, the SAT procedure should start for substation RTU.

Any other faulty case causes above described process repeating, up to the successful result of RTU software installation, RTU parameterization file uploading and appropriated SAT proceeding.

If such requirement is not realizable because of main grid conditions (e.g. energizing of primary equipment), the alternative solution of substation telematics system constructing could be possible. The separate RTU should be placed for each primary equipment part (bay based). In this way the volume of RTU SAT for every energizing stage of the substation project may be performed independently.

\section{CONCLUSION}

The main message of current article is the challenge to generate the new approach in relationship between TSO company and Contractor (Partner) for improving de facto independence of TSO company from manufacturers, for increasing of quality by Client's defined SAT and for bringing the know-how to the TSO company in part of RTU systems. It needs to do the cooperation work with Partners more tightly to achieve the above mentioned end target.

\section{REFERENCES}

[1] Official website of Elering OÜ [Online]. Available: http://www.elering.ee/index.php?id=102\&L=1. [Accessed May 17, 2010].

[2] Supply document of Elering OÜ, Requirements for Control and Monitoring.

[3] Standard EVS-EN (IEC harmonized) 60850-5-101/104

[4] Standard EVS-EN (IEC harmonized) 60850-5-103.

[5] "Sicam eRTU - Your entry to telecontrol technology with extended functionality", Official website of Siemens Corporation. [Online]. Available:

http://www.siemens.com.tr/i/assets/content/ogs/dagitim_yonu/s\%C4\%B 0cam_ertu.pdf\%20(229kb)\%20.pdf. [Accessed May 17, 2010].

[6] "RTU560/RTU211, Remote control based on new communication technologies," Official website of ABB Corporation. [Online]. Available:

http://www05.abb.com/global/scot/scot258.nsf/veritydisplay/01fcf64ac9 51d66bc1257590004db574/\$File/Xf3e2DEABB\%201456\%2009\%20E \%20RTU560_211\%20101209.pdf. [Accessed May 17, 2010].

[7] "Modern HMI system with SPRECON-V applications," Official website of Sprecher Automation GmbH company. [Online]. Available: http://www.sprecherautomation.com/upload/graphics/PDF/IKA/SPREC ON_V_84511021enZ.pdf. [Accessed May 17, 2010].

[8] "Type conformance test register of approved IEC 60870-5-10x tested equipment," Official website of KEMA company. [Online]. Available: http://www.kema.com/Images/COM110E\%20Type\%20conformance\%2 0Test\%20Register\%2060870-5.pdf. [Accessed May 17, 2010].

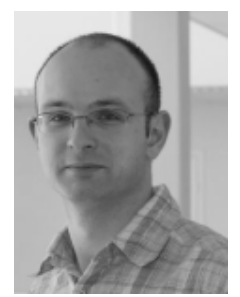

\section{Igor Dmitrenko}

Education.

1992-1997. Tallinn polytechnical school. Specialty of industrial and heating automation)

2000-2007. Tallinn University of Technology. Department of electrical drives and power electronics. Faculty of Power Engineering. Bachelor degree with documented master degree qualification

2007-present. Doctoral student of Tallinn University of

Technology. Faculty of Power Engineering. Estonia

Work experiences.

2001. Practice in $A M K G m b H \& C o . K G$. Production of industrial frequency converter. Germany

2003. Practice in SMN Elektrotechnik GmbH. Quality control of cable accessories production. Germany

1998-2006. Termest OÜ. Electrician, service specialist, service senior specialist. Supervision and service of unmanned water boiler and steam plants. SCADA system. Maintenance department. Tallinn. Estonia

2006-2007. Fortum Termest AS. Automation specialist. Unmanned water boiler and steam plants. Development department. Tallinn. Estonia

2007-present. Elering OÜ. Specialist of substation RTU systems. Technology department. Estonia 